\title{
Análisis Ontosemiótico de un Libro de Texto Colombiano de Educación Básica Primaria: el caso del Objeto Matemático Longitud
}

\author{
Cristian C. Fúneme Mateus \\ Leidy Julieth Linares Beltrán \\ Omaida Sepúlveda Delgado
}

\begin{abstract}
Resumen: En el documento se presentan los resultados más importantes de una investigación que tiene como objetivo: establecer el nivel de idoneidad didáctica de un libro de texto propuesto por el Ministerio de Educación Nacional de Colombia para abordar el objeto matemático longitud en el grado cuarto de educación básica primaria, bajo un enfoque cualitativo en el que se toman instrumentos del Enfoque Ontosemiótico del Conocimiento y la Instrucción Matemáticos (EOS), específicamente, la configuración de objetos primarios y los criterios de idoneidad didáctica. Además, se utiliza el significado referencial del objeto matemático longitud para el análisis de las situaciones propuestas en el texto. Lo anterior, permite concluir que el libro analizado tiene una idoneidad epistémica baja, debido a que presenta algunos conceptos de forma incorrecta o confusa y no considera la interacción entre los componentes del significado del objeto matemático.
\end{abstract}

Palabras clave: Idoneidad didáctica. Longitud. Enfoque Ontosemiótico. Libro de texto. Objeto matemático.

Cristian Camilo Fúneme Mateus Magister en Educación Matemática de la Universidad Pedagógica y Tecnológica de Colombia. Tunja, Boyacá, Colombia. https://orcid.org/0000-0002-9158-427X $\triangle$ cristian.funeme@uptc.edu.co

Leidy Julieth Linares Beltrán Magister en Didáctica de la Matemática de la Universidad Pedagógica y Tecnológica de Colombia. Tunja, Boyacá, Colombia. https://orcid.org/0000-0002-8220-9814

\leidy.linares@uptc.edu.co

Omaida Sepúlveda Delgado Doctora en Ciencias de la Educación y profesora de la Universidad Pedagógica y Tecnológica de Colombia. Tunja, Boyacá, Colombia. https://orcid.org/0000-0002-2950-8137

$\triangle$ omaida.sepulveda@uptc.edu.co

Recebido em 26/06/2021 Aceito em 04/09/2021 Publicado em 06/09/2021

\section{Ontosemiotic Analysis of a Colombian Textbook of Primary Basic Education: the case of the Mathematical Object Length}

Abstract: The paper presents the most important results of an investigation whose objective is to establish the level of didactic suitability of a textbook proposed by the Colombian Ministry of National Education to address the mathematical object length in the fourth grade of elementary basic education, under a qualitative approach in which instruments of the Ontosemiotic Approach to Mathematical Knowledge and Instruction (OSA) are used, specifically, the configuration of primary objects and the criteria of didactic suitability. In addition, the referential meaning of the mathematical object length is used for the analysis of the situations proposed in the text. The above allows concluding that the analyzed book has a low epistemic suitability, because it presents some concepts in an incorrect or confusing way and does not consider the interaction between the components of the meaning of the mathematical object.

Keywords: Didactic suitability. Length. Ontosemiotic Approach. Textbook. Mathematical object.

\section{Análise ontosemiótica de um Livro Didático colombiano da Educação Básica: o caso da Matemática Comprimento do objeto}

Resumo: 0 trabalho apresenta os resultados mais importantes de uma pesquisa que visa: estabelecer 0 nível de adequação didática de um livro didático proposto pelo Ministério da Educação Nacional da Colômbia para abordar a duração do objeto matemático na quarta série do ensino básico primário, sob uma abordagem qualitativa na qual os instrumentos da Abordagem Ontosemiótica do Conhecimento e Instrução Matemática (EOS) são tomados, especificamente, a configuração dos objetos primários e os critérios de adequação didática. Além disso, o significado referencial do comprimento do objeto matemático é usado 
para a análise das situações propostas no texto. 0 acima exposto nos permite concluir que o livro analisado tem uma baixa adequação epistêmica, pois apresenta alguns conceitos de forma incorreta ou confusa e não considera a interação entre os componentes do significado do objeto matemático.

Palavras-chave: Adequação didática. Comprimento. Enfoque Ontosemiótico. Livro Didático. Objecto matemático.

\section{Introducción}

En la búsqueda de estrategias y herramientas didácticas para la enseñanza y aprendizaje de las matemáticas, surgen los libros de texto escolares como elementos que conceden al estudiante autonomía en la construcción del conocimiento, proporcionándole explicaciones, ejemplos y ejercicios que le permiten explorar diferentes objetos matemáticos y sus características (FERNÁNDEZ y CABALLERO, 2007).

Estos instrumentos tienen una gran influencia en los currículos educativos, por ejemplo, en Colombia el uso del texto escolar se ve sometido a una serie de condicionamientos de orden ideológico, gubernamental, financiero y académico, aspectos que impactan en la labor del docente y en algunos casos dificultan los procesos de aprendizaje de los estudiantes (CORONADO et al., 2015).

En particular, en la última década los libros de texto propuestos para el área de matemáticas en Colombia se han desarrollado alrededor de los cinco tipos de pensamiento matemático descritos en los Estándares Básicos de Competencias del Ministerio de Educación de Colombia (MEN, 2006), específicamente los pensamientos numérico, espacial, métrico, aleatorio y variacional. En el caso del pensamiento métrico, corresponde a "la comprensión general que tiene una persona sobre las magnitudes y las cantidades, su medición y uso flexible de los sistemas métricos o medidas en diferentes situaciones" (MEN, 2006, p. 63).

Desde esta perspectiva, la longitud aparece como un tema fundamental en el desarrollo de la concepción de medida y en general en la construcción del sistema métrico decimal, esto lleva a que las diferentes editoriales lo presenten en sus textos escolares asociándolo a los sistemas de medida; por esta razón, es de vital importancia analizar si la manera en que lo hacen resulta didácticamente idónea para el trabajo dentro del aula y para el aprendizaje de los estudiantes.

Ahora bien, el análisis de un texto es amplio y complejo, por esta razón es necesario delimitar los aspectos a valorar. En este sentido, el Enfoque Ontosemiótico del Conocimiento y la Instrucción Matemáticos (EOS) (FONT et al., 2013; GODINO y BATANERO, 1994; GODINO et al., 2007) desde su postura epistemológica, antropológica y sociocultural de la matemática (GODINO, 2020) presenta los criterios de idoneidad didáctica que atienden a seis componentes: epistémico, 
cognitivo, interaccional, mediacional, emocional, y ecológico (GODINO et al., 2007), que permiten evaluar el grado de idoneidad con que un texto escolar aborda los objetos matemáticos.

Teniendo en cuenta las ideas expuestas, en el artículo se presentan los resultados relacionados con el análisis del grado de idoneidad didáctica que presenta el libro diseñado por el MEN (2017) para abordar el objeto matemático longitud en el grado cuarto de educación básica primaria. Se expone inicialmente el significado del objeto matemático desde un punto de vista histórico y epistemológico, luego se especifican cada una de las situaciones que se proponen en el texto y sus componentes del significado de referencia asociados. Finalmente, se realiza la valoración del libro a partir de los indicadores de idoneidad didáctica de la faceta epistémica (GODINO, 2013) y se presentan algunas conclusiones.

\section{Algunos elementos epistemológicos e históricos del significado de la longitud}

Aunque en la antigüedad los seres humanos no empleaban las matemáticas de manera formal, existía un uso implícito de ellas en sus diferentes labores diarias, siendo los babilonios los primeros en dar un paso cercano a su formalización al utilizar sus conocimientos de aritmética y algebra elemental, aplicados a longitudes y pesos, para realizar intercambios de moneda y mercancías (KLINE, 1992).

El trabajo con las medidas se afianzó en la antigua Grecia a través de la geometría, esto con los trabajos, por ejemplo, de Heródoto a quien se le atribuye la delimitación de los terrenos para distribuir las siembras en la ribera del río Nilo y de Tales de Mileto en el cálculo de las alturas de pirámides mediante congruencia de triángulos (D'AMORE; SBARAGLI, 2019). Aunque ninguno de ellos definió de forma concreta medida y longitud, dejaron entrever sus razonamientos hacía las mismas.

Uno de los primeros intentos por definir longitud fue dado por Pitágoras en su estudio de las fracciones, al expresar que "la longitud de una línea en una figura geométrica puede aproximarse tanto como queramos por un número racional" (STEWART, 2008, p. 26), en otras palabras, la longitud puede ser medida de forma constante y por ello debe ser racional. Esta incorporación del objeto longitud se hace presente también en los trabajos de Euclides, quien recurrió a ella en definiciones como: "una línea es una longitud sin anchura" (HEATH, 1908, p. 158).

El conjunto de ideas que se desarrollaron en torno a la longitud empezó a darle forma de magnitud continua, de hecho, Eudoxo (390-337 a. C.) expuso que "No se trataba de un número, sino de entidades tales como segmentos rectilíneos, ángulos, áreas, volúmenes, tiempo, etc., que 
podían variar, como si dijéramos, de una manera continua" (KLINE, 1992, p. 79), entendiéndola como una magnitud que puede medirse.

Así, al hablar de longitud se hace necesario abordar la medida, objeto en el que varios matemáticos han aportado notoriamente, entre ellos Henri Lebesgue, quien entre los años $1900 \mathrm{y}$ 1930 propuso la teoría de la medida a través de la idea central de conjuntos medibles, lo que le permitió extender la noción de medida a una función real positiva en los números reales.

Otro aspecto fundamental en el acercamiento al objeto longitud es el principio de continuidad, este fue propuesto por Poncelet (1822) al expresar que para cualquier figura, en una posición general en la que posee una o más relaciones o propiedades, métricas o descriptivas, al mantener estos mismos datos y variar la figura primitiva en grados insensibles o en un movimiento continuo, las propiedades y relaciones, encontradas para la primera seguirán siendo aplicables a los sucesivos estados resultantes, teniendo en cuenta las modificaciones particulares que pueden surgir como los cambios de signo, modificaciones que siempre serán fáciles de reconocer. En este sentido, ciertas características o propiedades de las figuras se mantienen invariantes bajo transformaciones como la traslación o la reflexión, tal es el caso de la longitud.

Con la idea inicial de que el principio de continuidad de Poncelet no era correcto, David Hilbert (1899) introdujo varios axiomas relacionados con la geometría y en especial con la continuidad, entre ellos se encuentra el de Arquimedianidad que permite fundamentar el proceso de medición a través de la comparación de la longitud de segmentos, definiendo de manera única un número (positivo) para cada segmento, el cual se identifica con la longitud de ese segmento (GIOVANNINI, 2013).

Así como la idea de medir ha estado presente en las preocupaciones del ámbito matemático, también lo ha hecho en la cotidianidad del ser humano, esto se puede evidenciar en aspectos como el establecimiento del sistema métrico de medidas en 1799, resultado del trabajo de matemáticos como Joseph-Louis Lagrange, Pierre Simon Laplace, Adrien Marie Legendre y Gaspard Monge en el comité de pesos y medidas de Francia. En dicho sistema se incluye al metro como unidad fundamental para la longitud (MERZBACH; BOYER, 2010).

\section{Significado referencial de longitud}

Los elementos históricos y epistemológicos mencionados, entre muchos otros, fueron adoptados y estructurados por Godino et al. (2002) para desarrollar un estudio didáctico profundo sobre la medida de las magnitudes, describiendo en particular que la longitud puede ser vista como 
un objeto matemático que tiene estructura de semimódulo al considerarlo bajo la adición y multiplicación usuales sobre el semianillo de los números naturales.

Lo mencionado hasta este punto, permite considerar principios de vital importancia para la caracterización del objeto longitud, algunos de carácter didáctico, tales como: percepción en la medición, principio de comparación, elección de instrumentos de medida, elección de unidades de medida y principio de conservación de la longitud; y otros relacionados con la estructura matemática: orden, suma y multiplicación de longitudes, la propiedad arquimediana y la transformación y equivalencias de las unidades de medida. Cada uno de estos componentes son tomados, adaptados y definidos en los siguientes párrafos a partir de la propuesta de Godino et al. (2002).

La percepción en la medición es el principio que reconoce la importancia de explorar las propiedades de los objetos concretos, para así, darle al estudiante el espacio donde pueda percibir que es aquello susceptible de ser medido en el mundo que lo rodea. Con esto, se hace explicito que no se debe dejar al azar este proceso de reconocimiento de la longitud y que es importante incorporarlo de manera sistemática en el aula.

En estrecha relación con la percepción, aparece la comparación entre objetos como un proceso natural, el cual no implica habilidades numéricas previas, por el contrario, emerge como una acción espontánea de los estudiantes al desear comparar aspectos como distancias, tamaño de objetos, estaturas, entre otros.

En este orden de ideas, al comparar objetos aparece la necesidad de tener patrones 0 referentes de medida. Es así, como surgen dos principios más, la elección de unidades y la elección de instrumentos de medida para la longitud, los cuales pueden ser de carácter estándar, como es el caso de uso de unidades del sistema internacional de medida y de instrumentos como el metro o la regla, o informales como los palmos, pasos o varas. Con lo anterior, aparece la sugerencia de permitir a los estudiantes desarrollar una primera experiencia perceptual que les ofrezca la posibilidad de relacionarlas con otras propiedades y conectarlas de un modo sistemático. Siendo fundamental en esta sistematización, el hacer conversiones entre unidades, como pasar centímetros a metros; siendo este el principio de Transformación y equivalencia.

En cuanto la conservación de la cantidad de longitud hace referencia a la característica propia de algunos cuerpos de no cambiar ante manipulaciones y cambios de situación. Este principio permite evaluar la capacidad de los estudiantes para evitar percepciones equivocadas de la longitud ante transformaciones como la reflexión, traslación, rotación o la modificación del tipo de segmento (curvo o recto). 
Ahora bien, el expresar que la cantidad de longitud puede ser vista como semimódulo con las operaciones suma y multiplicación de longitudes, permite contextualizar las operaciones en las que se puede trabajar dicho objeto matemático en educación básica primaria, incorporando, la aplicación de las propiedades distributiva, asociativa, conmutativa y de elemento neutro. Con esto, se establecen como componentes fundamentales en la construcción del objeto matemático longitud a la Suma, multiplicación y ordenación de longitudes.

Cuando se toma en consideración tanto a la multiplicación como la ordenación de longitudes se puede aplicar la Propiedad Arquimediana, de forma que al tener dos segmentos diferentes $\overline{A B}$ y $\overline{C D}$, esto es, con distinta medida de longitud, se puede encontrar un número $n \in$ $\mathbb{N}$ que, al multiplicarlo con la medida de uno de los segmentos, digamos $\overline{A B}$, arroja como resultado un segmento de mayor longitud que el otro, en este caso $\overline{C D}$.

Teniendo en cuenta lo anterior, para esta investigación se consideró que el significado referencial de la longitud puede ser alcanzado en las aulas de clase a partir de los principios y propiedades mencionadas que se resumen en la Figura 1.

Figura 1: Significado referencial de longitud en el contexto escolar

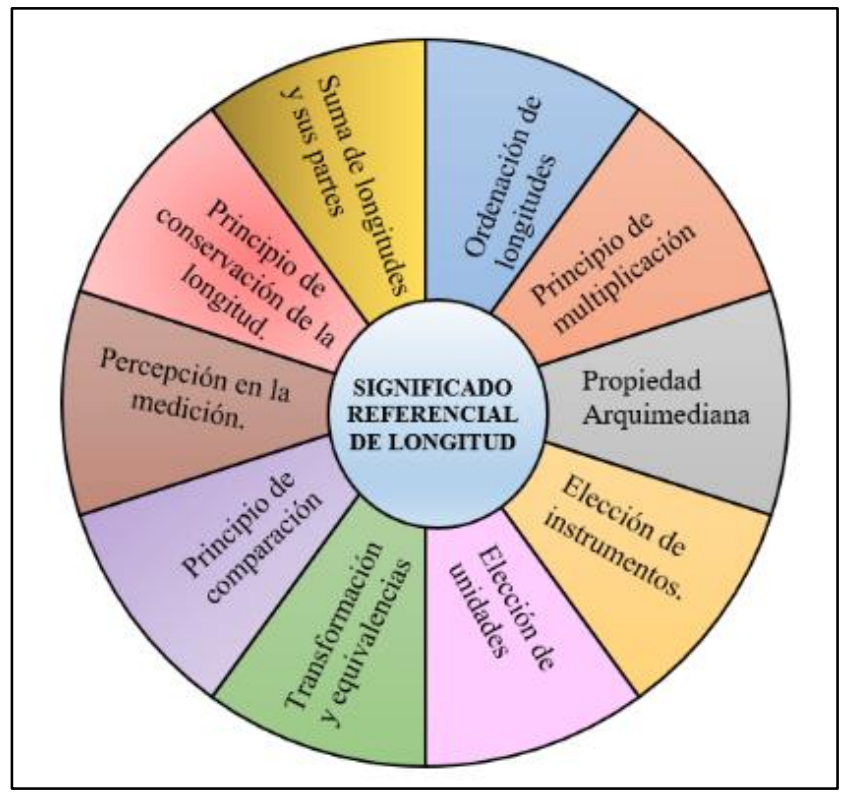

Fuente: Elaboración propia.

\section{El EOS y el análisis de textos escolares}

EI EOS es un enfoque que busca aportar en la construcción de un sistema teórico que permita abordar los problemas epistemológicos, ontológicos, semiótico-cognitivos y educativos implicados en la enseñanza y aprendizaje de las matemáticas (GODINO, 2020). En atención a lo anterior, dentro del EOS se ha propuesto una serie de elementos teóricos e instrumentos 
metodológicos de carácter antropológico y ontosemiótico, que permiten abordar la descripción, explicación, predicción, prescripción y valoración de los procesos de enseñanza y aprendizaje de las matemáticas, así como los factores que los condicionan (GODINO, 2018).

Al respecto, el presente artículo se enmarca dentro del problema educativo, en particular, en la optimización del proceso de instrucción, entendiendo que se abarca la relación entre los componentes del significado de referencia del objeto matemático longitud y la idoneidad de las situaciones que se presentan en un libro de texto para el aprendizaje de estudiantes de grado cuarto de educación básica primaria en Colombia. En este sentido, en el EOS se establece la idoneidad didáctica como un concepto sistémico de optimización de la instrucción matemática que reúne una serie de criterios que permiten, entre otras cosas, el análisis de recursos instruccionales (GODINO, 2013) que pueden ser aplicados teniendo en cuenta que "se deben adaptar e interpretar por parte del profesor, y se refieren a cada una de las facetas implicadas en los procesos de instrucción matemáticos: epistémica, ecológica, cognitiva, afectiva, interaccional y mediacional" (GODINO, 2020, p. 11).

La faceta epistémica comprende la relación que se puede establecer entre la diversidad de significados de los objetos matemáticos, la faceta cognitiva a la correspondencia entre los significados personales logrados por los estudiantes respecto de los pretendidos o implementados y la faceta interaccional a la organización y gestión de las tareas e interacciones que se puede establecer en el aula. En cuanto a la faceta mediacional se estudia si el uso de los diferentes recursos materiales y temporales resulta adecuado para potenciar el aprendizaje, en la faceta emocional se analiza el interés y motivación del estudiante por llevar a cabo el proceso de formación y la faceta ecológica aborda las relaciones entre los factores curriculares, políticos y económicos que condicionan los procesos de instrucción (GODINO, 2017).

Respecto al análisis de textos escolares, cuando se aborda el contenido propuesto en ellos se hace posible la valoración de la idoneidad epistémica, en donde se observan los objetos primarios: situaciones - problemas (aplicaciones extra-matemáticas, tareas, ejercicios, ...), elementos lingüísticos (términos, expresiones, notaciones, gráficos, ...), conceptos - definiciones (punto, número, segmento, ...), proposiciones (enunciados sobre conceptos, ...), procedimientos (algoritmos, operaciones, técnicas de cálculo, ...) y argumentos (enunciados usados para validar o explicar las proposiciones y procedimientos) (GODINO et al., 2007). A partir de estas entidades y de los componentes del significado de la longitud que se gestionan en las situaciones propuestas por el texto analizado, se procede a la valoración de los criterios de idoneidad.

Es importante aclarar que en el EOS los objetos matemáticos son considerados como "cualquier entidad que interviene de algún modo en la práctica o actividad matemática y que puede 
ser separado o individualizado" (GODINO, 2018, p.26). Además, la idea de significado es de tipo pragmático, reconociendo que el significado de las expresiones lingüísticas depende del contexto en que son utilizadas, de forma que el significado de los objetos matemáticos está sujeto a lo que hace el ser humano con ellos, es decir, a las prácticas personales o institucionales en las que emergen (GODINO, 2020).

\section{Descripción general del libro Vamos a Aprender Matemáticas 4}

El análisis del texto escolar Vamos a Aprender Matemáticas 4 (MEN, 2017) se realizó desde tres momentos: una descripción general de la estructura del texto en cuanto a contenidos matemáticos y la manera en que se estimula el desarrollo de los pensamientos numérico, espacial, métrico, aleatorio y variacional; codificación de objetos primarios en la situaciones que propone el libro para el aprendizaje de la longitud, identificando los componentes del significado de dicho objeto matemático en cada situación; y finalmente, la valoración de la idoneidad didáctica del texto.

Los libros del Ministerio de Educación Nacional de Colombia nacen en medio de un plan de acción gubernamental cuyo objetivo es hacer la nación más educada de América Latina en el 2025. El texto Vamos a Aprender Matemáticas 4 (MEN, 2017), que en adelante se nombrará como VAM, está distribuido en seis unidades se acuerdo a los cinco tipos de pensamiento matemático (MEN, 2006), a saber: el pensamiento numérico y sistemas numéricos se trabaja en la primera y segunda unidad, el pensamiento espacial y sistemas geométricos se presenta en la tercera unidad, el pensamiento métrico y sistemas de medidas en la cuarta unidad, el pensamiento aleatorio y sistemas de datos en la quinta unidad y finalmente el pensamiento variacional y sistemas algebraicos y analíticos en la sexta unidad.

Cada una de las unidades mencionadas cuenta con las siguientes subdivisiones: apertura de la unidad, presenta lo que el libro considera que el estudiante debe saber y lo que se busca que aprenda; ruta didáctica, que da desarrollo a cada uno de los contenidos y temas del libro; resolución de problemas que propone analizar y resolver varias situaciones utilizando diferentes estrategias, se da un ejemplo resuelto como guía y varios ejercicios para que el estudiante resuelva. Por último, se encuentra la evaluación del aprendizaje, en donde se proponen más ejercicios y preguntas relacionadas con la temática de la unidad.

Asimismo, en la presentación del libro se expone que algunas temáticas están relacionadas de forma transversal con tres proyectos: Educación para la sexualidad y ciudadanía, en la que se busca desarrollar competencias para ejercer, respetar y promover los derechos humanos que están presentes en las relaciones cotidianas; Educación Ambiental, que plantea 
actividades, ejemplos y situaciones con la intención de que el estudiante reflexione sobre las relaciones entre el ser humano y su entorno natural como proceso interactivo; y Estilos de Vida Saludable, en donde se tiene el propósito de que el estudiante aprenda a tomar decisiones sobre la salud y el bienestar físico, emocional e intelectual, tanto individual como colectivo.

Respecto al objeto de estudio de la investigación, magnitud - longitud, se ubica en el pensamiento métrico y sistemas de medidas en la cuarta unidad denominada Medición, las situaciones que se proponen en el texto se describen a continuación.

\section{Codificación de objetos primarios para el objeto longitud en el VAM}

Como resultado de la codificación deductiva de la unidad que el libro VAM señala para el aprendizaje de la longitud, se identificaron los objetos primarios que aparecen en la Tabla 1. En ella se hace alusión a tres definiciones, de las cuales dos son implícitas (D1 y D3), mientras que la otra es explicita (D3) y hace uso de la longitud para definir el perímetro.

Tabla 2: Codificación de objetos primarios

\begin{tabular}{|l|l|}
\hline \multicolumn{2}{|c|}{ Objetos primarios relacionados con la longitud } \\
\hline Definiciones & $\begin{array}{l}\text { D1. Unidad de medida: cantidad estandarizada de longitud. } \\
\text { D2. El perímetro de un polígono es la suma de las longitudes de todos sus lados. } \\
\text { D3. La longitud es una magnitud que permite medir objetos. }\end{array}$ \\
\hline Procedimientos & $\begin{array}{l}\text { P1. Reconocimiento de la cantidad de longitud en material concreto. } \\
\text { P2. Conversión entre unidades convencionales de medida de longitud, unidad } \\
\text { mayor a menor. }\end{array}$ \\
& $\begin{array}{l}\text { P3. Conversión entre unidades convencionales de medida de longitud, unidad } \\
\text { menor a mayor. }\end{array}$ \\
& $\begin{array}{l}\text { P4. Estimación de cantidad de medida. } \\
\text { P5. Adición de cantidades de longitud. }\end{array}$ \\
& $\begin{array}{l}\text { P6. División de cantidad de longitud entre número natural. } \\
\text { P7. Medición con unidades no convencionales. } \\
\text { P8. Conversión entre unidades convencionales de medida de longitud, unidad } \\
\text { menor a mayor (múltiplos de 10). } \\
\text { P9. Conversión entre unidades convencionales de medida de longitud, unidad } \\
\text { mayor a menor (submúltiplos de 10). } \\
\text { P10. Sustracción de cantidades de longitudes. }\end{array}$ \\
\hline Proposiciones & $\begin{array}{l}\text { Pr1. El perímetro se puede medir con patrones arbitrarios. } \\
\text { Pr2. El perímetro se puede medir con unidades de medida estándar. } \\
\text { Pr.3 Para medir el perímetro se parte de una longitud u, como patrón de medida. }\end{array}$ \\
\hline Argumentos & $\begin{array}{l}\text { A1. Ejemplos paradigmáticos de conversión de unidades. } \\
\text { A2. Ejemplificación de elección de unidades estandarizadas. } \\
\text { A3. Ejemplificación de cálculo de perímetro en situación paradigmática. } \\
\text { A4. Justificación de elección de una longitud como mayor a otra. }\end{array}$ \\
\hline
\end{tabular}




\begin{tabular}{|l|l|}
\hline Lenguaje & N. Lenguaje escrito mediante expresiones cotidianas y términos matemáticos. \\
& G. Representaciones gráficas. \\
& S. Expresiones que hacen uso del lenguaje matemático (símbolos, números, \\
& variables, etc.).
\end{tabular}
Fuente: Elaboración propia.

Antes de iniciar con el análisis de las situaciones en que emergen dichos objetos, es pertinente aclarar que los únicos procedimientos que aparecen como una explicación del texto para los estudiantes son P2, P3, P8 y P9, en ellos se recurre a gráficos para indicar la multiplicación y división por 10 (Figura 2) y sus múltiplos (Figura 3).

Figura 2: Procedimientos 2 y 3 para conversión de unidades de medida de longitud

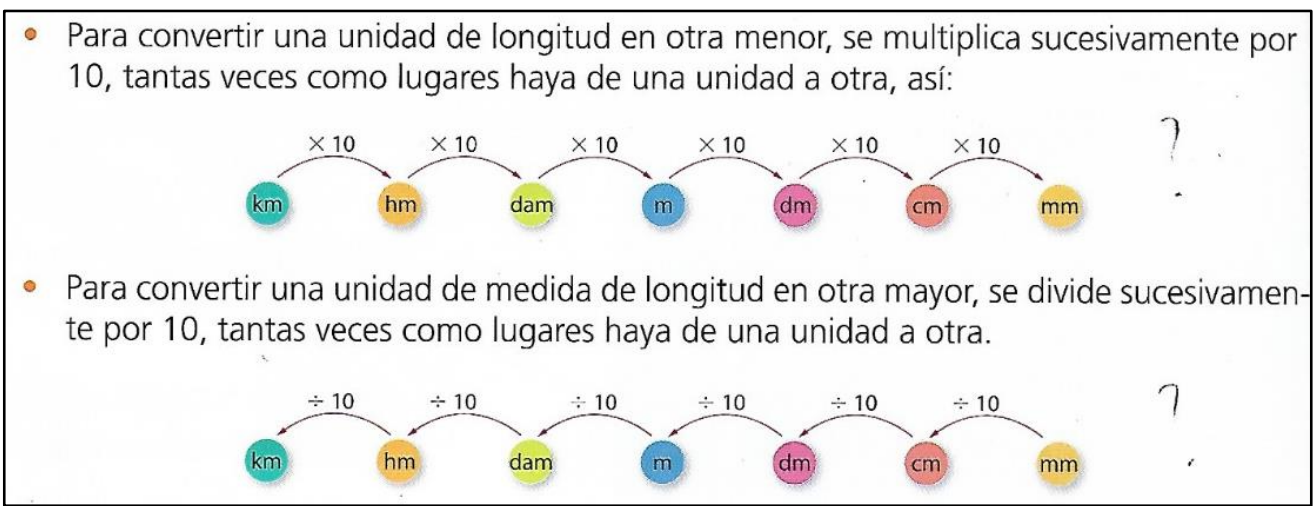

Fuente: Tomado de Men (2017, p. 100)

Figura 3: Procedimientos 8 y 9 para conversión de unidades de medida de longitud

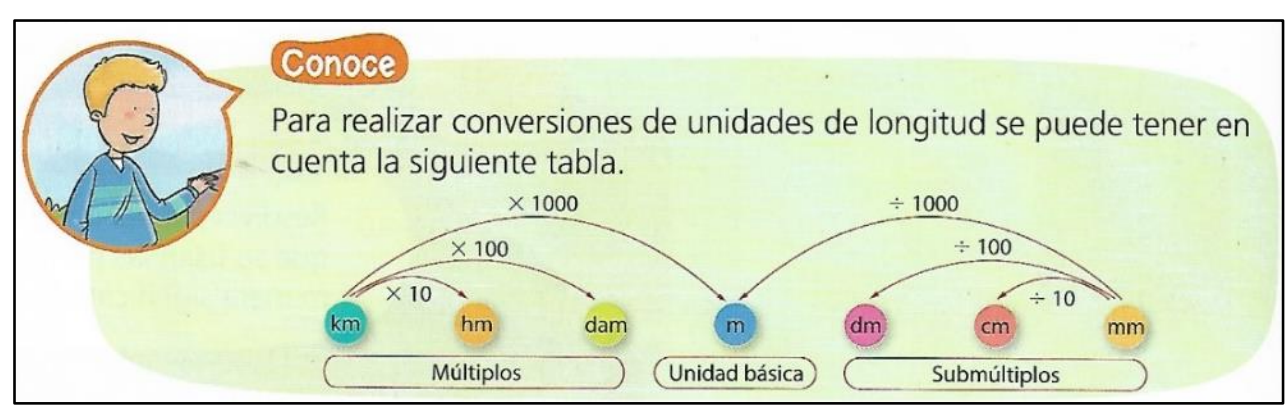

Fuente: Tomado de Men (2017, p. 100)

\section{Análisis de situaciones asociadas a la longitud}

El texto propone 21 situaciones en relación directa con la longitud, en esta sección se presenta un análisis detallado para cada una de ellas tomando en consideración los elementos del significado referencial de longitud (Figura 1) y los objetos primarios (Tabla 1). Dichas situaciones se dividieron en tres grupos teniendo en cuenta los principios a los que hacen mayor referencia; en el primero se agrupan las situaciones que hacen énfasis en la percepción de la medida y la elección de unidades; en el segundo, las situaciones centradas en la comparación y ordenación; y finalmente, el grupo de situaciones en que se da prioridad a la transformación de unidades de 
longitud y a los componentes de la estructura algebraica del objeto longitud (suma y multiplicación de cantidad de longitud).

\subsection{Situaciones relacionadas con la percepción de cantidad de longitud y elección de unidades}

Figura 4: Reconocimiento de unidades

Saberes previos

Toma una cinta métrica e identifica en ella un metro. ¿Cuántos decímetros, centímetros y milímetros hay en un metro?

Ve al cuaderno de trabajo.

Fuente: MEN (2017, p. 100)

La primera situación presentada en el texto (Figura 4) plantea el reconocimiento de unidades de medida. Esta situación se asocia al principio de la percepción de la longitud a través del reconocimiento de unidades de medida en material concreto, específicamente, haciendo uso de un lenguaje natural se trae a discusión la percepción que tiene el estudiante acerca del metro, decímetro, centímetro y milímetro. En lo anterior, sin hacer uso de proposiciones 0 argumentaciones, se espera que el estudiante desarrolle el procedimiento P1 (reconocimiento de la cantidad de longitud en material concreto). Con esta misma estructura se encuentran cuatro situaciones más, en la Figura 5 se resumen los objetos primarios relacionados con ellas.

Figura 5: Situaciones relacionadas con percepción en la medición

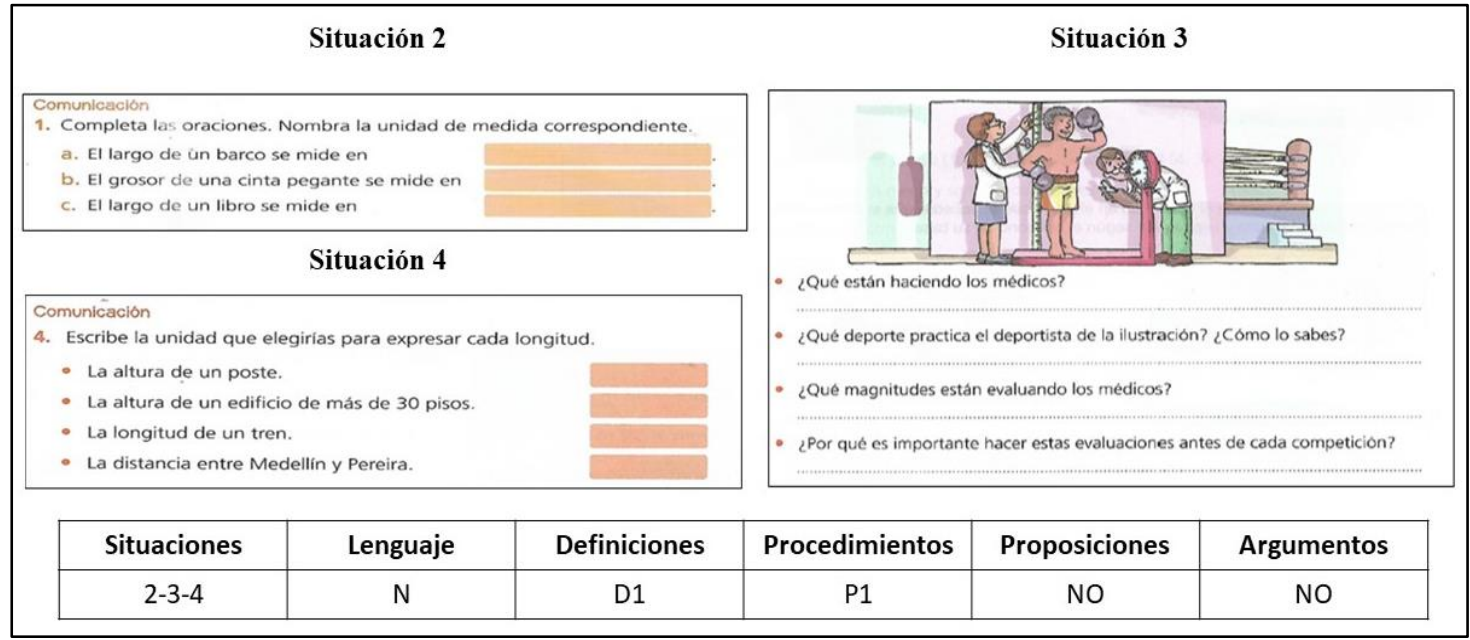

Fuente: Elaboración propia basada en situaciones de Men (2017)

\subsection{Situaciones relacionadas con la comparación y ordenación}

Las dos situaciones de la Figura 6 se relacionan con la definición de longitud desde el principio de comparación, el estudiante al abordarlas puede llegar a utilizar P1 (reconocimiento de la cantidad de longitud en material concreto), P4 (estimación de cantidad de medida) y P7 (medir con unidades no convencionales), no presentan proposiciones y utiliza un lenguaje verbal, gráfico 
y simbólico. Se espera que se generen argumentos por parte del alumno cuando diga por qué eligió una cantidad de longitud como mayor a otra en la situación 6.

Figura 6: Situaciones de comparación y ordenación de longitud

\begin{tabular}{|c|c|c|c|c|c|}
\hline \multicolumn{3}{|c|}{ Situación 5} & \multicolumn{3}{|c|}{ Situación 6} \\
\hline $\begin{array}{l}\text { Comunicacion } \\
\text { 1. Mide con palmos la longitu. } \\
\text { dibujo la medida obtenida. } \\
\text { a. }\end{array}$ & siguientes objetos re & de debajo de cada & \multicolumn{3}{|c|}{$\begin{array}{l}\text { Razonamiento } \\
\text { 3. Responde y comenta con uno de tus compañeros. } \\
\text { Sonia y Daniel midieron con pasos la longitud de una cuerda. Si un paso de Sonia } \\
\text { es más corto que el de Daniel, y Daniel midió cinco pasos, icómo es el resultado de } \\
\text { Sonia, mayor o menor que cinco pasos? ¿Por qué? }\end{array}$} \\
\hline Situaciones & Lenguaje & Definiciones & Procedimientos & Proposiciones & Argumentos \\
\hline $5-6$ & N-G-S & D3 & P1-P4-P7 & NO & A4 \\
\hline
\end{tabular}

Fuente: Elaboración propia basada en situaciones de Men (2017)

\subsection{Situaciones que dan prioridad a la estructura algebraica y las transformaciones}

Figura 7: Situaciones con transformación y equivalencias, suma y multiplicación de longitudes

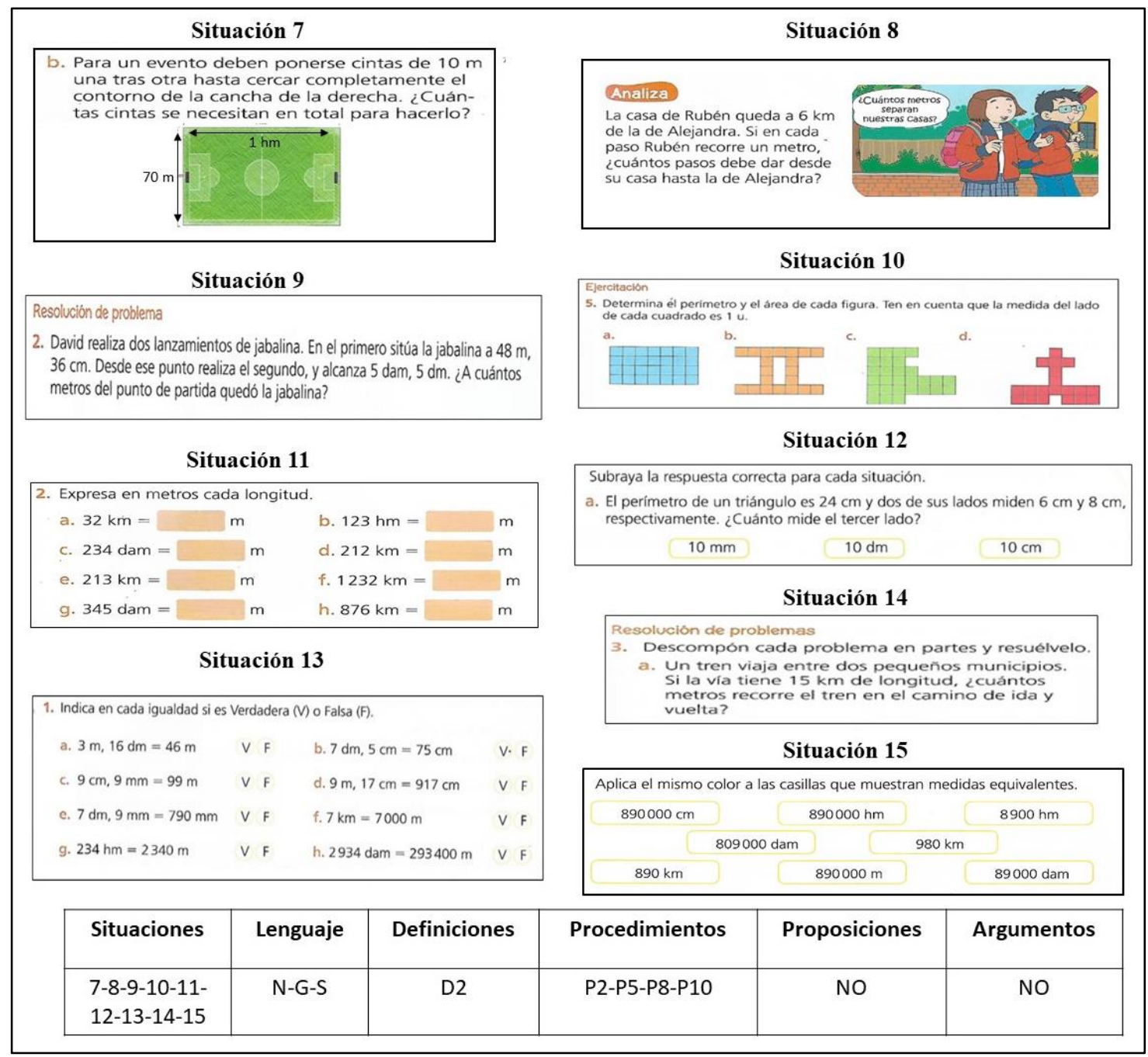

Fuente: Elaboración propia basada en situaciones de Men (2017) 
La situación 7, expuesta en la Figura 7, se presenta en un lenguaje verbal, gráfico y simbólico (Números y notación de unidades de medida) y con la solución de ella se puede llegar a emplear los procedimientos P2-P3-P8-P9 (conversión entre unidades convencionales de medida de longitud) y P5-P10 (adición y sustracción de cantidad de longitudes). En ella se reconocen los principios de transformación y equivalencias, suma y multiplicación de longitudes. Aspecto similar al de las demás situaciones que se presentan en la Figura 7.

Dentro de este mismo tipo de gestión del significado de la longitud, se encuentran las 6 situaciones que se presentan en la Figura 8, en donde aparecen los procesos de P5-P10 (adición y sustracción de cantidad de longitudes), esto sin incorporar transformación en las unidades de medida y como ha sido reiterativo en la propuesta del texto, sin argumentos o proposiciones.

Figura 8: Situaciones con suma y multiplicación de longitudes

\begin{tabular}{|c|c|c|c|c|c|}
\hline \multicolumn{3}{|c|}{ Situación 16} & \multicolumn{3}{|c|}{ Situación 17} \\
\hline $\begin{array}{l}\text { Ejercitación } \\
\text { 1. Ten en cuenta las longitudes de } \\
\text { a. }\end{array}$ & $\begin{array}{l}\text { ados de cada poti } \\
\qquad \text { b. }\end{array}$ & Calcula sus perimetros. & \multicolumn{3}{|c|}{$\begin{array}{l}\text { 1. Deduce las medidas que faltan en cada figura y halla su perimetro. } \\
\text { a. }\end{array}$} \\
\hline \multicolumn{3}{|c|}{ Situación 18} & \multicolumn{3}{|c|}{ Situación 19} \\
\hline \multicolumn{3}{|c|}{$\begin{array}{l}\text { Analiza } \\
\text { Agustin destinó parte de su } \\
\text { granja para la siembra de papa, } \\
\text { maiz y zanahoria, como se } \\
\text { observa en el dibujo. ¿Cuátos } \\
\text { metros de alambre necesita para } \\
\text { cercar todos los cultivos? }\end{array}$} & \multicolumn{3}{|c|}{$\begin{array}{l}\text { Sigue un método y soluciona estos problemas. } \\
\text { a. Antonio conistruyó un cuadrado de } 28 \mathrm{~cm} \\
\text { de perímetro. ¿Cuánto mide cada lado? } \\
\text { b. Dibuja un cuadrado, un hexágono y un } \\
\text { nonágono cuyos perímetros sean iguales al } \\
\text { perímetro de la siguiente figura. } \\
12 \mathrm{~cm}\end{array}$} \\
\hline \multicolumn{3}{|c|}{ Situación 20} & \multicolumn{3}{|c|}{ Situación 21} \\
\hline \multicolumn{3}{|c|}{$\begin{array}{l}\text { b. La longitud del lado de una mesa cuadrada mide } 90 \mathrm{~cm} \text {. ¿Cuál es su } \\
\text { perímetro? }\end{array}$} & \multicolumn{3}{|c|}{$\begin{array}{l}\text { Razonamiento } \\
\text { 2. Dibuja un poligono que cumpla la condición dada en cada literal. } \\
\text { a. Cuadrado de } 12 \mathrm{~cm} \text { de perimetro. b. Rectángulo de } 18 \mathrm{~cm} \text { de perimetro. }\end{array}$} \\
\hline Situaciones & Lenguaje & Definiciones & Procedimientos & Proposiciones & Argumentos \\
\hline $\begin{array}{c}16-17-18-19- \\
20-21\end{array}$ & N-G-S & D2 & P2-P5-P8-P10 & NO & NO \\
\hline
\end{tabular}

Fuente: Elaboración propia basada en situaciones de Men (2017)

\section{Valoración de la idoneidad epistémica}

Para establecer la valoración, se toman en consideración los componentes e indicadores propuestos por Godino (2013), empleando cinco niveles de evaluación para la idoneidad alcanzada en cada uno de ellos: nula (0), si el texto no presenta situaciones que cumplan con los indicadores; baja (1), si presenta alguna situación que dé cumplimiento a alguno de los indicadores; media (2), si propone varias situaciones que cumplen (cada una) con alguno de los 
indicadores; alta (3), si presenta varias situaciones que cumplen (cada una) con uno de los indicadores propuestos; o total (4), si el texto aborda por completo y en diversas situaciones, cada uno de los indicadores. Es importante aclarar, que los resultados que se presentan se basan exclusivamente en lo expuesto por el libro y no incluyen análisis de la gestión del libro en el aula.

\subsection{Componente 1. Situaciones-problemas}

Criterio 1. Se presenta una muestra representativa y articulada de situaciones de contextualización, ejercitación y aplicación.

De acuerdo con el análisis de las situaciones presentadas por el libro VAM, se determina que la muestra de situaciones de ejercitación, aplicación y contextualización corresponde al nivel de idoneidad media, pues abordan: mediciones de objetos con unidades no convencionales, el procedimiento de conversión de unidades y el desarrollo de ejercicios con un contexto artificial; sin embargo, no se presentan situaciones de contextualización en la que se incorporen unidades e instrumentos convencionales.

De hecho, las situaciones que se presentan como problemas utilizan contextos con datos teóricos y no obtenidos por el estudiante y para resolverlos basta con la reiteración de los algoritmos de conversión y las operaciones de adición, multiplicación y división, limitando la posibilidad de utilizar los problemas como actividades de contextualización, análisis, comunicación y generalización, elementos que dentro del EOS se consideran fundamentales para entender la resolución de problemas como eje central de la emergencia de los objetos matemáticos (GODINO, 2013).

Criterio 2. Se proponen situaciones de generación de problemas (problematización).

Para este criterio se considera que el libro VAM tiene una idoneidad nula, dado que no existe ninguna situación dentro del texto, en donde se promueva la formulación de problemas por parte de los estudiantes en relación con la longitud. Más aún, no existen espacios donde el estudiante pueda problematizar la información que se presenta en las situaciones que debe resolver.

\subsection{Componente 2. Situaciones-problemas}

Criterio 3. Uso de diferentes modos de expresión matemática (verbal, gráfica, simbólica...), traducciones y conversiones entre las mismas.

En las situaciones propuestas por el libro VAM se encuentran diferentes modos de representación matemática (lenguaje natural, gráfico y simbólico), de forma que los estudiantes deben hacer uso de diversas expresiones para dar sus soluciones. Sin embargo, se percibe un mayor énfasis en la expresión simbólica, dado que solo en las situaciones 19 y 21 se busca que 
los estudiantes realicen gráficas y únicamente en la 3 y 6 se solicita la expresión del lenguaje natural, las demás (17 situaciones) requieren exclusivamente de valores numéricos e indicar las unidades de medida de la longitud. Por lo expuesto, se considera que la idoneidad en este criterio es media.

Criterio 4. Nivel del lenguaje adecuado a los niños a que se dirige.

En este criterio la idoneidad resulta alta, entendiendo que las expresiones que se emplean en su mayoría son de carácter informal y en las ocasiones que se recurre al lenguaje matemático corresponde al nivel de formación de los estudiantes. Además, el texto se compone esencialmente de situaciones a desarrollar, la presencia de definiciones, proposiciones y argumentos es mínima, por lo cual es escaso el espacio para encontrar dificultades en el lenguaje empleado, aspectos que de hecho no permiten considerar el lenguaje como idóneo pues los procedimientos de explicación del texto solo indican pasos a seguir, pero carecen de argumentación para que el estudiante los comprenda.

Criterio 5. Se proponen situaciones de expresión matemática e interpretación.

Este último criterio correspondiente al componente 1 es de idoneidad media en el texto analizado, entendiendo que 10 situaciones (situaciones número $3,6,7,8,9,12,14,18,19$ y 21) son planteadas de manera en que el estudiante debe hacer interpretaciones de la información que se le otorga, para luego expresarse matemáticamente respecto a ellas. Un aspecto que no permite una valoración más alta es que las situaciones solo se relacionan con el proceso de conversión de unidades y con el diseño de polígonos, no hay variaciones adicionales, de forma que llegan a ser reiterativas en lo que debe interpretar el estudiante.

\subsection{Componente 3. Reglas}

Criterio 6. Las definiciones y procedimientos son claros y correctos, y están adaptados al nivel educativo al que se dirigen.

Se encuentran tres definiciones y tres procedimientos que se pretenden desarrollar. La primera definición es la de longitud, la cual no es trabajada explícitamente si no que se espera que el estudiante la desarrolle a partir de la solución de las diferentes situaciones. La segunda nuevamente es implícita y se refiere a la unidad de medida. La tercera definición hace referencia al perímetro a través de la expresión: "El perímetro de un polígono es la suma de las longitudes de todos sus lados o de otra forma, la medida de su contorno. Este se puede medir con patrones arbitrarios o con unidades de medida estándar" (MEN, 2017, p. 102).

Si bien, esta definición se puede entender como correcta no se considera del todo clara y adaptada de forma adecuada para el grado cuarto, ya que, en primera medida, al mencionar que 
es la suma de las longitudes de todos sus lados, no se menciona la necesidad de la homogeneidad de las unidades de medida, sin embargo, en las respuestas que deben dar los estudiantes si se hace necesaria esa homogeneidad. Además, se hace uso de términos como contorno, patrones arbitrarios y unidades de medida estándar de los cuales no se presentan definiciones ni se consideran dentro de las situaciones de conocimientos previos.

Adicionalmente, en la explicación que ofrece el texto para la conversión de unidades se presentan dos alternativas, inicialmente se muestran los procedimientos P2 y P3 en donde se considera la conversión de unidades a través de la multiplicación o división, respectivamente, sucesivamente por 10; mientras que, en los procedimientos P8 y P9 se expresa la posibilidad de multiplicar o dividir por múltiplos de 10. Sin embargo, para ejemplificar P2 se indica que: "se multiplica sucesivamente por 10 , tantas veces como lugares haya de una unidad a otra" (MEN, 2017, p. 100) y luego se presenta la operación que no corresponde a dicha expresión (Figura 9), relacionada con $\mathrm{P} 8$, pero dicho procedimiento no se ha presentado cuando se aborda esta ejemplificación. En atención a lo expuesto respecto al criterio 6 , se considera que la idoneidad del libro es baja.

Figura 9: Situaciones con suma y multiplicación de longitudes

$$
6 \mathrm{~km}=6 \times 1000 \mathrm{~m}=6000 \mathrm{~m} \text {. }
$$

Fuente: Tomada de Men (2017, p. 100)

Criterio 7. Se presentan los enunciados y procedimientos fundamentales del tema para el nivel educativo dado.

En este criterio la idoneidad encontrada es baja, considerando que las situaciones e información que se maneja en el texto carece de enunciados que ayuden al aprendizaje, por el contrario, se centra en cuatro procedimientos que tienen la misma intención, conversión de unidades, omitiendo aspectos de la longitud como la estimación, la comparación, el uso de instrumentos de medida y el error.

Criterio 8. Se proponen situaciones donde los alumnos tengan que generar o negociar definiciones proposiciones o procedimientos.

Dado que son pocas las situaciones de respuesta abierta y que el texto solo presenta como procedimiento a desarrollar la conversión de unidades, se considera que la idoneidad en este criterio es baja. Además, la naturaleza misma de las situaciones limita la posibilidad de negociación de las definiciones, procedimientos y proposiciones, pues en ellas no se encuentran diversidad de estrategias de solución e incluso de solución, la mayoría de ellas se reducen a seleccionar datos, hacer conversión a una unidad de longitud determinada y formular la respuesta. 
Más aún, en ninguna situación el estudiante debe expresar en sus palabras definiciones o proposiciones y tampoco se ofrecen situaciones en que los estudiantes propongan sus propios procedimientos para realizar mediciones de cantidad de longitud o establecer equivalencias entre sus unidades de medida.

\subsection{Componente 4. Argumentos}

Criterio 9. Las explicaciones, comprobaciones y demostraciones son adecuadas al nivel educativo a que se dirigen.

Se establece que las explicaciones sobre la longitud no son adecuadas para el grado cuarto debido a que no presentan argumentos del por qué se hace uso de ciertos algoritmos, solo hay una ejemplificación y su intención únicamente es apoyar la explicación dada, pero no se da al estudiante información de cómo surgen las operaciones realizadas o como a través de ellas se relacionan las unidades de medida. Adicional o la anterior, no se encuentra la promoción de la comprobación de resultados o de situaciones relacionadas con la demostración de propiedades como la conservación de la cantidad de longitud. De esta forma, se establece que en este criterio la idoneidad del texto es baja.

Criterio 10. Se promueven situaciones donde el alumno tenga que argumentar.

De las 21 situaciones que propone el libro VAM, solo en las situaciones 3 y 6 se solicita al estudiante argumentar su solución, y solo en la situación 3 esa argumentación tiene relación con la longitud, en las demás situaciones solo se pide un resultado numérico o de elección de unidad de medida de la longitud, de manera que se establece que respecto a este criterio la idoneidad del texto es baja.

\subsection{Componente 5. Relaciones}

Criterio 11. Los objetos matemáticos (problemas, definiciones, proposiciones, etc.) se relacionan y conectan entre sí.

En el libro de texto se encuentra la presencia de conexiones con los objetos distancia, perímetro y área; sin embargo, las situaciones que permiten esta conexión no pasan de ser algorítmicas, omitiéndose argumentaciones y construcciones que permitan al estudiante reconocer por qué están relacionadas. Es decir, se observa un énfasis en las relaciones entre definiciones y procedimientos, pero no con los demás objetos primarios, lo que lleva a una valoración de idoneidad baja en este criterio.

Criterio 12. Se identifican y articulan los diversos significados de los objetos que intervienen en las prácticas. 
Dado el tipo de objetos primarios identificados en el texto (Tabla 1) y los componentes del significado referencial de la longitud que se identificaron en cada situación, se establece que en el libro VAM no existe una articulación clara del significado de la longitud, por el contrario, solo se encuentra gestión de algoritmos para la conversión de unidades, omitiendo o minimizando significados necesarios para la comprensión de los mismos, como lo que se entiende por unidad de medida, unidad básica de medida, múltiplo y submúltiplos de una unidad de medida.

A partir de la valoración dada para cada uno de los criterios de la idoneidad epistémica se encuentra que el libro VAM tiene una idoneidad didáctica baja en esta faceta (Tabla 2).

Tabla 2: Valoración de componentes e indicadores de idoneidad epistémica

\begin{tabular}{|c|c|c|}
\hline Componente & Indicador & VAM \\
\hline $\begin{array}{l}\text { Situaciones } \\
\text { problemas }\end{array}$ & $\begin{array}{l}\text { - Se presenta una muestra representativa y articulada de } \\
\text { situaciones de contextualización, ejercitación y aplicación. } \\
\text { - Se proponen situaciones de generación de problemas } \\
\text { (problematización). }\end{array}$ & 2 \\
\hline Lenguaje & $\begin{array}{l}\text { - Uso de diferentes modos de expresión matemática (verbal, } \\
\text { gráfica, simbólica...), traducciones y conversiones entre los } \\
\text { mismas. } \\
\text { - Nivel del lenguaje adecuado a los niños a que se dirige. } \\
\text { - Se proponen situaciones de expresión matemática e } \\
\text { interpretación. }\end{array}$ & $\begin{array}{l}3 \\
2\end{array}$ \\
\hline Reglas & $\begin{array}{l}\text { - } \quad \text { Las definiciones y procedimientos son claros y correctos, y están } \\
\text { adaptados al nivel educativo al que se dirigen. } \\
\text { - Se presentan los enunciados y procedimientos fundamentales } \\
\text { del tema para el nivel educativo dado. } \\
\text { - Se proponen situaciones donde los alumnos tengan que generar } \\
\text { o negociar definiciones proposiciones o procedimientos. }\end{array}$ & 1 \\
\hline Argumentos & $\begin{array}{l}\text { - Las explicaciones, comprobaciones y demostraciones son } \\
\text { adecuadas al nivel educativo a que se dirigen. } \\
\text { - Se promueven situaciones donde el alumno tenga que } \\
\text { argumentar. }\end{array}$ & $\begin{array}{l}1 \\
1\end{array}$ \\
\hline Relaciones & $\begin{array}{l}\text { - Los objetos matemáticos (problemas, definiciones, } \\
\text { proposiciones, etc.) se relacionan y conectan entre sí. } \\
\text { - Se identifican y articulan los diversos significados de los objetos } \\
\text { que intervienen en las prácticas. }\end{array}$ & 1 \\
\hline & Valoración Promedio & 1 \\
\hline
\end{tabular}

\section{Consideraciones adicionales sobre facetas del EOS}

Considerando que los criterios de idoneidad de las facetas restantes propuestas por el EOS (cognitiva, afectiva, interaccional, mediacional y ecología) requieren de un análisis de la implementación del libro de texto, observando las interacciones entre docentes, estudiantes y conocimiento, a continuación, se presentan algunas observaciones respecto a ellas más no su 
valoración, pues esta investigación solo contempla la información que se encuentra en el texto y no el uso por parte de profesores.

Para iniciar, respecto a la idoneidad cognitiva se considera que no se presentan explícitamente los conocimientos que el estudiante requiere para el desarrollo de las situaciones que proponen como alternativa para el aprendizaje. Además, en dichas situaciones se enfatiza en suma de cantidad de longitudes, principio de conservación y transformación y equivalencias, dándole menor importancia a factores como la percepción y comparación de longitudes, importantes en los niveles iniciales de formación al permitir acercar al estudiante al objeto matemático, esto sin mencionar que en las 21 situaciones se minimiza la importancia de gestionar el orden de las longitudes, principio de la multiplicación y la elección de instrumentos de medida.

El segundo aspecto por considerar es el de adaptaciones curriculares a las diferencias individuales, al realizar una revisión de este componente se establece que el texto no presenta actividades de ampliación sobre el objeto longitud, en cambio presenta varias situaciones que buscan que el estudiante refuerce los algoritmos. De hecho, en la forma en que se desarrolla el proceso de evaluación del aprendizaje se detectan falencias en lo propuesto por el texto, pues no se encuentran diversos modos de evaluación, comprensión, representación y aplicación de habilidades.

Por otra parte, al explorar la propuesta del texto escolar a partir de los componentes de idoneidad afectiva, se encuentra que las actividades difícilmente serán de alto interés para alumnos de grado cuarto debido a que son pocas las situaciones que muestran la utilidad de la longitud y no se hace uso de la cotidianidad de los estudiantes en ellas. Asimismo, no se tiene en cuenta atributos de la matemática como la estética y la precisión.

Además, el trabajo del estudiante es primordialmente individual, con poca oportunidad de diálogo y argumentación donde se pueda promover el respeto y tolerancia por la opinión de los demás, esta carencia genera que tampoco se encuentren espacios de gestión para la autoestima de los estudiantes.

En lo referente a la idoneidad interaccional se establece que las situaciones planteadas en el texto producen una escasa oportunidad de un diálogo entre profesor y estudiantes del cual surjan preguntas que amplíen el conocimiento de los estudiantes, desarrollen capacidad argumentativa, propositiva y de expresión a través de diversas formas de representación.

De manera similar ocurre con el diálogo entre estudiantes, pues las actividades son en su mayoría individuales y limitan la gestión de un proceso que les permita a los alumnos convencerse de la validez de las afirmaciones, ideas, proposiciones y argumentos que tienen respecto al objeto longitud. Esto genera perdida de espacios de inclusión y respeto a las diferencias. 
Esta interacción entre estudiante, conocimiento y profesor que se propone en el texto, minimiza la importancia que tiene el contexto para generar una comunicación significativa entre ellos, esto se afirma considerando que no hay actividades que permitan reflexionar al estudiante sobre la presencia de la longitud en su entorno para plantear problemas y posibles soluciones. Tampoco se presentan situaciones significativas de ejemplos y contraejemplos que muestren porque es conveniente o no elegir ciertas unidades o instrumentos, lo cual podría ayudar en la gestión de procesos de investigación y planteamientos de conjeturas.

Sobre la idoneidad mediacional, en lo que refiere al uso de material manipulativo, el texto propone mínimamente y de manera superficial, actividades que permiten a los estudiantes manipular material concreto, de hecho, se plantean situaciones de establecer cantidad de longitud en representaciones gráficas de elementos que podrían ser medidos por los estudiantes directamente en objetos que se encuentran en sus entornos.

En cuanto el tiempo utilizado para el desarrollo de las actividades, el texto no presenta una orientación para el docente de cómo podrían distribuirse las actividades en diversas sesiones o en cuáles de las situaciones se puede requerir mayor tiempo y acompañamiento del profesor, solo se limita a presentar una serie de ejercicios y actividades.

Para finalizar, en los componentes de la idoneidad ecológica se observa que el contenido del texto corresponde a las directrices curriculares colombianas, pero presenta falencias en las actividades y evaluación (esto fue discutido en las idoneidades previas). Al considerar el desarrollo del pensamiento crítico, un aspecto que no se ha discutido y que juega un rol fundamental en esta idoneidad, se considera como ausente en el texto al no encontrarse aspectos como los valores democráticos, interacción y conservación del medio ambiente, estilo de vida, sostenibilidad, entre muchos otros temas que promueven una comprensión de la realidad a partir del objeto matemático abordado.

Tampoco se tienen en cuenta sectores económicos de Colombia, de ellos se podrían tomar situaciones de aprendizaje contextualizadas y significativas. La única sección que busca atender al pensamiento crítico es el proyecto educación para la sexualidad y ciudadanía en el que se retoma la educación matemática critica desde la visión de Skovsmose (1999), sin embargo, en dicho proyecto la longitud no se vincula.

\section{Conclusiones y recomendaciones}

La construcción del significado referencial del objeto matemático longitud se llevó a cabo mediante un rastreo histórico, encontrando que en el acercamiento a él se ha hecho necesario 
tomar en consideración la suma y multiplicación de longitudes con sus propiedades, el orden de las longitudes, el principio de percepción, comparación y conservación, la propiedad arquimediana, la transformación y equivalencias, así como la elección de instrumentos y unidades. Cada uno de estos componentes, permitió caracterizar la idoneidad epistémica, de lo que se deduce que en el texto no se le da la misma importancia a cada uno de estos componentes, razón por la cual el estudiante podría tener una construcción parcial de este significado.

Además, se encontró que, a pesar de que en general está diseñado bajo los lineamientos curriculares que propone el Ministerio de Educación Nacional Colombiano, el texto presenta una idoneidad didáctica baja al presentar falencias en cuanto a las situaciones que se proponen, ya que no llevan a la argumentación por parte de los estudiantes.

Ahora bien, al identificar los significados, objetos y procedimientos propuestos en el libro de texto, se encuentran situaciones reiterativas basadas en algoritmos y carentes de innovación para los estudiantes; ya que, no se encuentran situaciones que motiven a la indagación o que incluyan de manera significativa el contexto.

En síntesis, la investigación deja en evidencia que en el libro de texto sigue predominando la metodología conductista con énfasis en los procesos algorítmicos. Por tal motivo, se resalta que es fundamental el papel del docente como mediador en el proceso de aprendizaje de sus estudiantes, siendo importante su capacidad para vincular estrategias y actividades complementarias a las propuestas por el texto que se le otorga para trabajar en el aula.

Finalmente, la investigación resalta la importancia de analizar los elementos que son llevados al aula como recurso docente, debido a que no todos cumplen con la idoneidad didáctica para presentar ciertos objetos matemáticos. En este caso, se evidencia la necesidad de analizar la forma en que los textos escolares dan a conocer la longitud, pues es un objeto que se menciona en el contexto educativo sin reconocer su aspecto global, reduciendo su valor e importancia.

\section{Referencias}

CORONADO, Jorge; CASTAÑEDA, Dora; TIQUE, Carlos. La pertinencia del uso del texto escolar: características, condiciones y restricciones. In VELÁSQUEZ, D.; LÓPEZ, R. (Eds.). EI texto escolar: investigaciones sobre sus perspectivas y uso en la ciudad de Bogotá. Bogotá: Kimpres S.A.S, 2015. p. 37-64.

D'AMORE, Bruno; SBARAGLI, Silvia. La matematica e la sua storia. Vol. III. Dal Rinascimento al XVIII secolo. Bari: Dedalo, 2019.

FERNÁNDEZ, María; CABALLERO, Presentación. El libro de texto como objeto de estudio y recurso didáctico para el aprendizaje: fortalezas y debilidades. Revista electrónica interuniversitaria de formación del profesorado, v. 20, n. 1, p. 201-217, 2017. 
FONT, Vicenç; GODINO, Juan; GALLARDO, Jesús. The emergence of objects from mathematical practices. Educational Studies in Mathematics, v. 82, p. 97-124, 2013.

GIOVANNINI, Eduardo. Completitud y continuidad en Fundamentos de la geometría de Hilbert: acerca del Vollständigkeitsaxiom. Theoria, n. 76, p. 139-163, 2013.

GODINO, Juan. Indicadores de la idoneidad didáctica de procesos de enseñanza y aprendizaje de las matemáticas. Cuadernos de Investigación y Formación en Educación Matemática, $\mathrm{n}$. 11, p. 111-132, 2013.

GODINO, Juan. Construyendo un sistema modular e inclusivo de herramientas teóricas para la educación matemática. In CONTRERAS, J.; ARTEAGA, P.; CAÑADAS, G.; GEA, M.; GIACOMONE, B.; LÓPEZ, M. (Eds.). Actas del Segundo Congreso International Virtual sobre el Enfoque Ontosemiótico del Conocimiento y la Instrucción Matemáticos, 2017. Disponible en http://enfoqueontosemiotico.ugr.es/civeos.html.

GODINO, Juan. Bases semióticas, antropológicas y cognitivas del enfoque Ontosemiótico en educación matemática, 2018. Disponible en http://enfoqueontosemiotico.ugr.es/JDGodino_bases_sac_EOS.pdf. Acceso el 22 de junio de 2021.

GODINO, Juan. El enfoque ontosemiótico:Implicaciones sobre el carácter prescriptivo de la didáctica. Revista Chilena de Educación Matemática, v. 12, n. 2, p. 3-15, 2020.

GODINO, Juan; BATANERO, Carmen. Significado personal e institucional de los objetos matemáticos. Recherches en Didactiques des Mathématiques, v. 14, n. 3, p. 325-355, 1994.

GODINO, Juan; BATANERO, Carmen; FONT, Vicenç. The ontosemiotic approach to research in mathematics education. ZDM. The International Journal on Mathematics Education, v. 39, n. 1-2, p. 127-135, 2007.

GODINO, Juan; BATANERO, Carmen; ROA, Rafael. Medida de magnitudes y su didáctica para maestros. Granada: Universidad de Granada, 2002.

HEATH, Thomas. The thirteen books of the elements. Cambridge: The University Press, 1908.

HILBERT, David. Grundlagen der Geometrie. Göttingen, 1899.

KLINE, Morris. El pensamiento matemático de la antigüedad a nuestros días. Madrid: Alianza editorial, 1992.

MEN. Estándares Básicos de Competencias. Bogotá: Ministerio de Educación Nacional, 2006.

MEN. Vamos a aprender Matemáticas 4. Bogotá: Ediciones SM S.A, 2017.

MERZBACH, Uta; BOYER, Carl. A history of Mathematics. Third edition. New Jersey: John Wiley \& Sons Inc., 2010.

PONCELET, Jean V. Traité des propriétés projectives des figures. Paris, 1822.

STEWART, lan. Historia de las matemáticas en los últimos 10.000 años. Barcelona: Crítica. 2008. 
SKOVSMOSE, Ole. Hacia una filosofía de la educación matemática crítica. Bogotá: Una empresa docente, Universidad de los Andes, 1999. 